\title{
Distributed and Antagonistic Contributions of Ongoing Activity Fluctuations to Auditory Stimulus Detection
}

\author{
Sepideh Sadaghiani, ${ }^{1,2,3,4}$ Guido Hesselmann, ${ }^{1,2,4}$ and Andreas Kleinschmidt ${ }^{1,2,4}$ \\ ${ }^{1}$ Unité 562, Cognitive Neuroimaging, Institut National de la Santé et de la Recherche Médicale and ${ }^{2}$ Commissariat à l'Energie Atomique, Direction des \\ Sciences du Vivant, Institut d'Imagerie Biomédicale, NeuroSpin, 91191 Gif-sur-Yvette Cedex, France, ${ }^{3}$ Graduate School of Neural and Behavioural Sciences, \\ International Max Planck Research School, University of Tübingen, 72074 Tübingen, Germany, and ${ }^{4}$ Université Paris-Sud, 91405 Orsay, France
}

Recent studies have shown that ongoing activity fluctuations influence trial-by-trial perception of identical stimuli. Some brain systems seem to bias toward better perceptual performance and others toward worse. We tested whether these observations generalize to another as of yet unassessed sensory modality, audition, and a nonspatial but memory-dependent paradigm. In a sparse event-related functional magnetic resonance imaging design, we investigated detection of auditory near-threshold stimuli as a function of prestimulus baseline activity in early auditory cortex as well as several distributed networks that were defined on the basis of resting state functional connectivity. In accord with previous studies, hits were associated with higher prestimulus activity in related early sensory cortex as well as in a system comprising anterior insula, anterior cingulate, and thalamus, which other studies have related to processing salience and maintaining task set. In contrast to previous studies, however, higher prestimulus activity in the so-called dorsal attention system of frontal and parietal cortex biased toward misses, whereas higher activity in the so-called default mode network that includes posterior cingulate and precuneus biased toward hits. These results contradict a simple dichotomic view on the function of these two latter brain systems where higher ongoing activity in the dorsal attention network would facilitate perceptual performance, and higher activity in the default mode network would deteriorate perceptual performance. Instead, we show that the way in which ongoing activity fluctuations impact on perception depends on the specific sensory (i.e., nonspatial) and cognitive (i.e., mnemonic) context that is relevant.

\section{Introduction}

Despite its ubiquitous prevalence, spontaneous activity has conceptually long been considered technical or biological "noise" and has been discarded as unexplained variance when estimating average evoked neural responses to an externally imposed paradigm. Yet, ongoing activity fluctuations contribute to variability of evoked responses (Arieli et al., 1996) and impact on perceptual performance (Linkenkaer-Hansen et al., 2001; Boly et al., 2007; Hesselmann et al., 2008a,b). These effects range from focal observations in functionally specialized regions to distributed patterns involving areas that are less specifically related to task demands.

Perceptual performance is affected by variations of preceding ongoing (Boly et al., 2007) or evoked (Li et al., 2007; Eichele et al., 2008) activity in neuroanatomical systems that resemble those networks which functional neuroimaging studies have defined on the basis of coherent spontaneous fluctuations during resting wakefulness (Fox and Raichle, 2007). One of these so-called resting-state networks that comprises posterior cingulate and precuneus, the temporoparietal junction, and medial prefrontal

\footnotetext{
Received May 29, 2009; revised Sept. 2, 2009; accepted Sept. 21, 2009.

This work was funded by the Agence Nationale de la Recherche (SPONTACT; France). S.S. is supported by the Friedrich-Ebert Foundation (Germany). This fMRI experiment was part of a general research program on functional neuroimaging of the human brain, which was sponsored by the Atomic Energy Commission (Denis Le Bihan). We thank Evelyn Eger for helpful comments on data analysis.

Correspondence should be addressed to Sepideh Sadaghiani, NeuroSpin/CEA/SAC/DSV/I2BM, Bât 145, Point Courrier 156, F-91191 Gif-sur-Yvette, France. E-mail: sepideh.sadaghiani@gmail.com. D0I:10.1523/JNEUROSCI.2592-09.2009

Copyright $\odot 2009$ Society for Neuroscience $\quad$ 0270-6474/09/2913410-08\$15.00/0
}

regions will stereotypically deactivate during a typical sensorimotor or cognitive task. This task-negative behavior correlates with the degree of functional challenge (McKiernan et al., 2003; Greicius and Menon, 2004; Mason et al., 2007; Singh and Fawcett, 2008) and has been taken as a possible sign of suspending a default mode of brain function (Gusnard and Raichle, 2001). Another network of bilateral frontal and parietal regions is involved in attentional control, and of all resting-state networks, its activity is least correlated with that in the default-mode network (Fox et al., 2005; Fransson, 2005). Together with other systems, this network usually shows task-positive behavior, i.e., activation when subjects are engaged in a paradigm.

These observations could indicate that higher ongoing activity in sensory and attentional brain systems facilitates perceptual performance, whereas higher activity in regions of the default mode network deteriorates perceptual performance. The present study sought to explore several related issues. Our starting hypothesis was that where and how ongoing activity impacts on perceptual performance is strongly context dependent. Our own previous studies with perceptual decisions on supraliminal but ambiguous visual stimuli identified effects from ongoing activity only in those brain regions where functional specialization matched the perceptual feature to be judged (Hesselmann et al., 2008a,b). We hypothesized that for detection of near-threshold stimuli, effects would be unveiled not only in specifically related sensory cortex but also in other more generic brain systems as suggested by a somatosensory study (Boly et al., 2007). We also sought to test whether previous observations in the visual 
(Hesselmann et al., 2008a,b) and sensorimotor system (Boly et al., 2007; Fox et al., 2007) generalize to the auditory modality where evidence is so far lacking. We hence chose an auditory task that relies more on recognition and less on spatial orienting than somatosensory and visual perception and focused on the relation of stimulus detection and prestimulus activity in auditory cortex as well as the aforementioned task-positive and task-negative systems.

\section{Materials and Methods}

Subjects, imaging data acquisition, and paradigm. Twelve right-handed normal-hearing subjects (two female; ages, 19-30) gave written informed consent before participation in imaging on a 3T magnetic resonance imaging (MRI) whole-body scanner (Tim-Trio; Siemens). The study received ethics committee approval by the authorities responsible for our institution. One subject reported to have fallen asleep in one session and was thus excluded from analysis. Anatomical imaging used a T1-weighted magnetization-prepared rapid acquisition gradient echo sequence $[176$ slices, repetition time $(\mathrm{TR})=2300 \mathrm{~ms}$, echo time $(\mathrm{TE})=$ $4.18 \mathrm{~ms}$, filed of view (FOV) 256 , voxel size $1 \times 1 \times 1 \mathrm{~mm}$ ). Functional imaging used a $2^{*}$-weighted gradient-echo, echo-planar-imaging sequence ( 25 slices, TR $=1500 \mathrm{~ms}, \mathrm{TE}=30 \mathrm{~ms}$, FOV 192, voxel size $3 \times$ $3 \times 3 \mathrm{~mm}$ ). Stimulus presentation and response recording used the Cogent Toolbox (John Romaya, Vision Lab, UCL; www.vislab.ucl.ac.uk) for MATLAB (Mathworks) and sound delivery a commercially available MR-compatible system (MR Confon).

We acquired in the same subjects at an earlier occasion 820 volumes of task-free "resting state" data (with closed, blind-folded eyes) and then, on a later occasion, experimental sessions of 820 volumes each during which subjects were exposed to sparse near-threshold auditory stimuli and performed an auditory detection task. An additional passive localizer run for defining auditorily responsive brain regions was acquired after the main experiment. This 81 volume run consisted of three $20 \mathrm{~s}$ blocks of repetitive stimulus presentation with $0.5 \mathrm{~s}$ interstimulus intervals (ISI) at clearly audible volume separated by $15 \mathrm{~s}$ baseline epochs.

The auditory stimulus was a $500 \mathrm{~ms}$ noise burst with its frequency band modulated at $2 \mathrm{~Hz}$ (from white noise to a narrower band of $0-5$ $\mathrm{kHz}$ and back to white noise). Subjects were blindfolded and instructed to report as quickly and accurately as possible by a right-hand key press whenever they heard the target sound despite scanner's background noise. In a first $6.5 \mathrm{~min}$ run, which was not analyzed, we determined each subject's auditory threshold using a simple staircase procedure. We refer to auditory threshold as the signal level for which the probability of detection is $50 \%$. The staircase procedure started out at a clearly audible volume and applied a simple up-down rule (von Békésy, 1960) with 25 trials and interstimulus intervals randomized between 2.5 and $5 \mathrm{~s}$. The signal level of the last 6 trials was averaged to yield an estimate of subjects' threshold. Next, each subject performed two and some subjects three experimental runs of $20 \mathrm{~min}$ duration. On each run, target stimuli were presented at individual threshold (periliminal stimuli) on 36 trials and at a fixed suprathreshold level on 4 "catch" trials. ISIs ranged unpredictably from 20 to $40 \mathrm{~s}$, with each specific ISI used only once. Before each run, the target stimulus was played a few times at suprathreshold volume for (re)memorization, and subjects were informed that in most of the trials the target sound would be played at a barely perceptible level. If within $1.5 \mathrm{~s}$ of stimulus onset a key was pressed, this trial was counted as a hit, if not as a miss. All other key presses were classified as false alarms.

Standard analysis of functional magnetic resonance imaging data. We used statistical parametric mapping (SPM5, Wellcome Department of Imaging Neuroscience, UK; www.fil.ion.ucl.ac.uk) for image preprocessing (realignment, coregistration, normalization to MNI stereotactic space, spatial smoothing with a 5 and $6 \mathrm{~mm}$ full-width at half-maximum isotropic Gaussian kernel for single-subject and group analyses, respectively) and estimation of general linear models with a high-pass filter of $1 / 128 \mathrm{~Hz}$ and realignment parameters as nuisance covariates.

Regressors for event-related analysis were obtained by convolving for each condition unit impulse time series with a canonical hemodynamic response function. The statistical model included the four events hits, misses, false alarms, and catch trials. Timing of false alarm events (response in the absence of an external stimulus) was defined based on the subject's mean reaction time in hits. For each subject, we estimated condition-specific effects using a general linear model, then created contrast images and entered these into a second-level one-sample $t$ test.

Unless otherwise stated, we report activations of this standard analysis at $p<0.05$ corrected at the cluster level for multiple comparisons using an auxiliary (uncorrected) voxel threshold of $p<0.0001$. This auxiliary threshold defines the spatial extent of activated clusters, which form the basis of our (corrected) inference. Results are rendered onto an inflated average brain as provided by FreeSurfer (CorTechs Labs; http://surfer.nmr.mgh.harvard.edu) with the help of SPM SurfRend toolbox (http://spmsurfrend.sourceforge.net).

Resting state functional connectivity analysis. We used a 20 min taskfree session recorded in each of the subjects on an earlier occasion to analyze resting-state functional connectivity ( $\mathrm{rs}-\mathrm{fc}$ ). We thereby defined the default mode network as well as two other large-scale networks, one related to spatial attention and another to "task set maintenance" (Dosenbach et al., 2006, 2007). In the context of our study, we preferred to label the latter system as an "intrinsic alertness" network for the following reasons: This label allowed us to pay tribute to results involving this network in previous functional neuroimaging studies probing auditory intrinsic alertness (Sturm et al., 2004) and to the role that alertness has been assigned in performance fluctuations during long-lasting auditory detection tasks (Makeig and Inlow, 1993). Seed regions for calculation of rs-fc networks were the gray-matter voxels in a sphere of $10 \mathrm{~mm}$ radius centered on stereotactic coordinates reported in three metaanalyses by other laboratories: (1) Posterior cingulate cortex (PCC)/precuneus $(-5,-49,40)$ and medial prefrontal cortex (MPFC)/ventral anterior cingulated cortex (vACC) $(-1,-47,4)$ for the default mode network (Fox et al., 2005); (2) right intraparietal sulcus (IPS) (27, -58 , $49)$ and right frontal eye fields (FEF) $(24,-13,51)$ for the dorsal spatial attention system (Fox et al., 2006); and (3) right anterior insula $(36,16,4)$ and right anterior thalamus $(10,-15,8)$ for the intrinsic alertness system. While coordinates for the latter system were hence derived from studies revolving around task set maintenance (Dosenbach et al., 2007), it should be pointed out that those as well as our results partially overlapped with results from studies that used slightly different seed regions in the anterior insula and that provided other functional interpretations for their findings (Seeley et al., 2007; Eckert et al., 2009). We, therefore, preferred to apply a label to this network that was intuitively plausible within the context of our own present study.

For each of six seed regions, the high-pass $(1 / 128 \mathrm{~Hz})$-filtered signal time course was averaged across all respective voxels and used as a regressor of interest in a separate general linear model. Imaging data were preprocessed as described above. In addition to the same nuisance variables as in the activation experiment, we also included the global signal of three separate brain compartments (all white matter voxels, all gray matter voxels, and all CSF voxels) as covariates of no interest. Contrast images corresponding to each regressor were created for each subject and entered into a second-level one-sample $t$ test for each seed region. Again, maps were rendered onto a canonical average brain provided in FreeSurfer.

Definition of regions of interest. We defined several regions of interest (ROIs) from which we extracted time course data estimated in a finite impulse model procedure. Voxels responding to the auditory stimulus were defined on a subject-by subject basis in two steps. First, at the group level, the contrast periliminal stimuli (i.e., hits and misses) $>$ baseline $(p<0.001)$ was masked by the passive auditory localizer contrast at $p<$ 0.001 . A spherical search space of $10 \mathrm{~mm}$ was defined around the peak of the periHeschl clusters with the highest $z$ score [right hemisphere: (42, $-6,-12), z=4.14,94$ voxels and left hemisphere: $(-42,-18,-6), z=$ $3.9,23$ voxels]. Next, for each subject's corresponding first-level contrast, all voxels within this search space were selected that passed a lenient threshold ( $p<0.05$, uncorrected).

Further ROIs were defined from the rs-fc maps (see above). For every subject, default mode, spatial attention, and intrinsic alertness networks were defined as those voxels whose time course significantly correlated at $p<0.001$ (uncorrected) with that of both respective seed regions. The 
resulting subject-specific rs-fc networks were then further constrained by masking with the corresponding group result. These group masks were defined at a second level of analysis as the overlap of corresponding pairs of rs-fc networks from either seed region at threshold $p<0.005$, uncorrected, and cluster extent $>200$ voxels. The right and left middle temporal complex $(\mathrm{MT}+)$ clusters (31 and 14 voxels in the conjunction analysis) were also included in the group mask of the spatial attention system, because they have been considered one of the system's core constituents in cognitive studies (Corbetta and Shulman, 2002) and in rs-fc studies (Fox et al., 2006). In the group statistical map of the intrinsic alertness system, bilateral anterior insula and anterior thalamus merged into a single big cluster. To enable separate investigation of these regions, the map was masked by anatomically defined masks of insula and thalamus, respectively (WFU PickAtlas 2.4, Wake Forest University School of Medicine; http://www.fmri.wfubmc.edu/cms/software).

For each subject, the resulting networks as a whole and each of their clusters in isolation were used as ROI for time course extraction.

Statistical analysis of functional magnetic resonance imaging signal time courses. After the aforementioned spatial preprocessing, we used a temporal high-pass filter with very low cutoff $(1 / 1000 \mathrm{~Hz})$ and no prewhitening. This ensured linear drift removal, a common practice in many related studies to deal with technical noise sources (Golland et al., 2007; Hesselmann et al., 2008a,b), while minimizing interference with low frequency brain activity fluctuations. A finite impulse response (FIR) model was applied using 24 peristimulus stick functions $(\times 1.5 \mathrm{~s}$ bins $)$ for each of the four conditions, hits, misses, false alarms, and catch trials. Nuisance covariates included the realignment parameters.

The estimated time course was then averaged across voxels of specific ROIs. Condition-dependent time courses were indistinguishable for corresponding regions of the two hemispheres and therefore collapsed. For each ROI, a paired $t$ test of "perceptual outcome" was performed for the hemodynamic signal at the immediate prestimulus time point $0 \mathrm{~s}$. This time point was chosen because it is the latest epoch that is with certainty not yet contaminated by stimulus-evoked activity. Accordingly, it has proven informative to predict perceptual outcome in earlier related studies (Hesselmann et al., 2008a,b). Based on our previous observations, we tested one-tailed in the auditory ROI, where we hypothesized prestimulus signal to be larger for detected stimuli and two-tailed in all other ROIs. For display purposes, but not statistical analyses, signal time courses were temporally smoothed with a $(1,2,1)$ kernel.

The FIR model was also used to generate a map of prestimulus effects on auditory detection by contrasting parameter estimates for hit and miss trials at time point $0 \mathrm{~s}$. The corresponding contrast images were entered into a second-level one-sample $t$ test. We report results of this analysis at $p<0.05$ cluster-level significance after applying an auxiliary voxel-level threshold of $p<0.01$ and extent threshold $>50$ voxels.

\section{Results}

A group of 11 subjects performed a detection task on auditory stimuli presented at their individually determined detection threshold in a very sparse event-related functional MRI (fMRI) paradigm with highly variable ISI ranging from 20 to $40 \mathrm{~s}$.

\section{Behavioral findings}

Overall, the stimulus was detected in slightly more than half of periliminal trials $(62.2 \pm 17 \%$ hits $)$. The individual hit-miss ratio was largely consistent across separate sessions, with the exception of one session each in two of the subjects with $94.4 \%$ hits (i.e., only two misses). Subjects' accuracy in detecting the rare supraliminal catch stimuli was $93.2 \pm 0.1 \%$. Reaction times were $788 \pm$ $102 \mathrm{~ms}$ for detected periliminal stimuli (hits) and $578 \pm 142 \mathrm{~ms}$ for detected catch trials. Subjects reported a highly variable number of false alarms. Many sessions had no false alarms at all, although each subject reported at least one false alarm. The median of false alarms per session (within-subject average across sessions) was median $=4.5 \pm$ interquartile range $=6.9$.

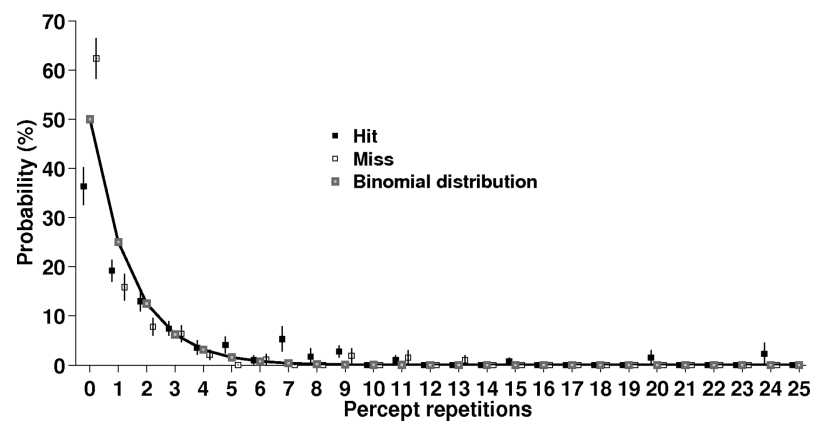

Figure 1. Distribution of percept repetitions. The incidence of repetitions for hits and misses is well approximated by a binomial distribution (goodness-of-fit $R^{2}=0.93$ for hits, $R^{2}=0.83$ for misses).

The incidence of percept repetitions was well approximated by a binomial distribution indicating approximately stochastic stimulus detection and hence independence of successive trials (Fig. 1). The probability for different perceptual outcomes in two immediately successive trials (i.e., "zero repetition") reflected the slight overall bias for hits. Finally, there was no bias for misses to occur after shorter or longer ISIs than hits. The length of prestimulus ISIs was indistinguishable (paired $t$ test $p=0.6$ ) between hits $(30.6 \pm 0.7 \mathrm{~s})$ and misses $(30.3 \pm 1 \mathrm{~s})$.

\section{Imaging results}

Figure 2 shows statistical parametric maps of evoked responses. Hits versus baseline involved distributed activation in a large set of cortical and subcortical areas summarized in supplemental Table 1, available at www.jneurosci.org as supplemental material. Misses versus baseline did not yield significant activation in these maps ( $p<0.001$, uncorrected), nor did misses versus hits. A direct comparison of hits versus misses revealed an activation pattern very similar to hits versus baseline with an additional activation in the cerebellum (supplemental Table 1, available at www.jneurosci.org as supplemental material).

\section{Signal time course of early auditory cortex}

Figure 3 shows the time course from the bilateral auditory region. Voxels in auditory cortex responding to the periliminal stimulation (hit and miss > baseline) were selected for time course extraction on a subject-by-subject basis. They were restricted to a search sphere that was defined at group level by masking activation foci of periliminal stimulation with a mask obtained from a passive auditory localizer session. The Talairach coordinates [42, $-6,-12$ and $-42,-18,-6]$ of the search sphere are in good agreement with cytoarchitectonic (Morosan et al., 2001) and functional (Scott and Johnsrude, 2003) descriptions of Heschl's gyrus and presumably include parts of primary auditory cortex. Based on previous results (Hesselmann et al., 2008a,b), we tested the immediate prestimulus time point $0 \mathrm{~s}$ for percept-dependent differences in BOLD signal time course. As hypothesized, we found slightly but significantly higher prestimulus baseline signal in hits than in misses $\left(t_{(1,10)}=2.21, p=0.025\right.$ one-tailed $)$.

\section{Signal time course of functional networks}

We next investigated the signal time course in a set of distributed networks and their constituent regions. The spatial patterns of rs-fc networks that we obtained here are in good agreement with previous studies (Greicius et al., 2003; Fox et al., 2005; Fransson, 2005; Dosenbach et al., 2007; Seeley et al., 2007). Figure 4 shows each network as defined from an independent resting-state ses- 


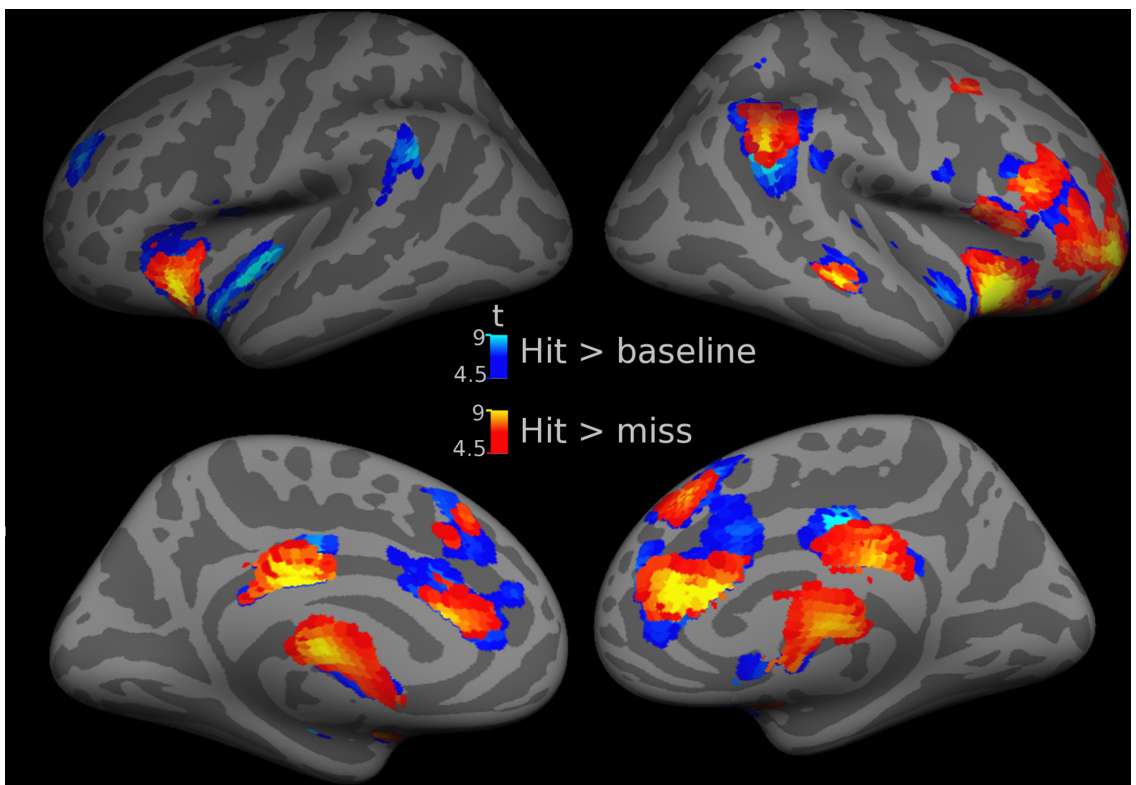

Figure 2. Spatial distribution of evoked cortical responses during successful stimulus detection. Activations evoked in hit trials versus baseline are shown in cold colors (for details, compare supplemental Table 1, available at www.jneurosci.org as supplemental material). A direct comparison of greater responses during hits than misses (warm colors) revealed a very similar activation pattern. Threshold height $p<0.05$ corrected at the cluster level using an auxiliary (uncorrected) voxel threshold of $p<0.0001$. Group results $(n=11)$ are superimposed onto the lateral and medial aspects of an inflated cortical surface of a canonical average brain.

\section{bilateral auditory cortex}
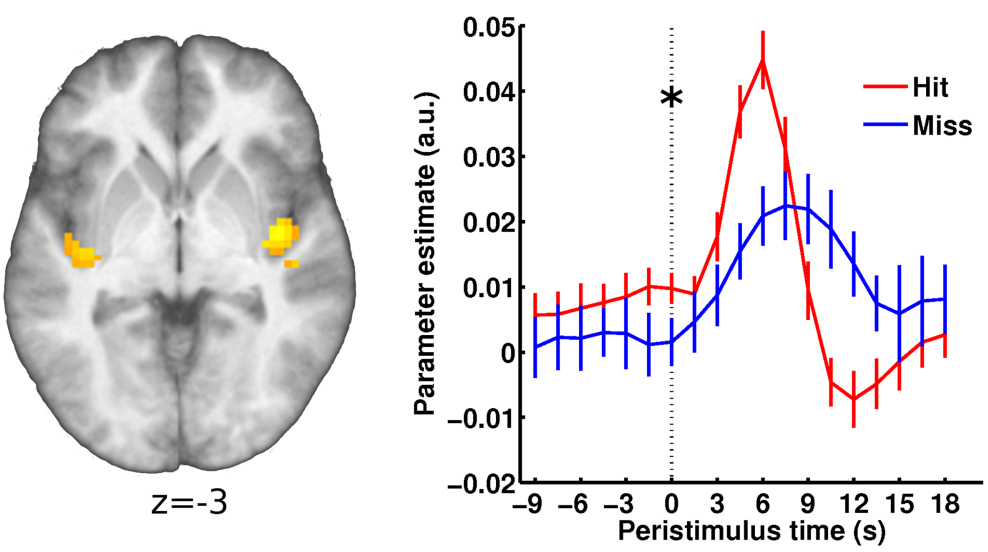

Figure 3. Prestimulus fMRI time courses from bilateral auditory cortex. Left, Map of activation evoked by the near-threshold stimulus (independent of percept) assessed in a group analysis. This map served as the basis for subject-by-subject definition of the auditory ROI (shown on the group's average brain; threshold height $p<0.001$ uncorrected; see Materials and Methods for details). Right, In accord with our previous findings, we tested the effect of prestimulus activity at time point $0 \mathrm{~s}$ and found significantly higher activity preceding hits than misses (as indicated by an asterisk). Error bars indicate \pm SEM.

sion by rs-fc analysis. It also shows the corresponding signal time courses in the auditory detection sessions. For each system as a whole and for each of its constituent regions in isolation, these signal time courses were tested for differences at time point $0 \mathrm{~s}$ as a function of perceptual outcome.

The dorsal attention system showed higher prestimulus activity preceding misses than hits $\left(t_{(1,10)}=3.82, p<0.005\right)$. On a region-by-region level, this effect was significant in IPS $\left(t_{(1,10)}=\right.$ 4.21, $p<0.005)$ and MT $+\left(t_{(1,10)}=2.23, p<0.05\right)$ but not in FEF. Interestingly, task-positive behavior was preserved in the evoked responses during hit trials, whereas misses showed a blunted pattern, if any stimulus-locked response at all. In con- trast, hits were preceded by significantly higher prestimulus baseline activity in the default mode network than misses (whole system: $\left.t_{(1,10)}=2.43, p=0.036\right)$. This effect was driven by the $\mathrm{PCC} /$ precuneus component $\left(t_{(1,10)}=2.61, p=0.026\right)$, whereas the baseline signal difference did not reach significance in MPFC/vACC or lateral parietal cortex. Again, tasknegative behavior was conserved across all regions but only in hit trials, and it appeared fairly late in relation to stimulus timing. Moreover, in the precuneus, the heightened prestimulus signal in hit trials evolved into an early but stimulus-locked task-positive response that was absent in miss trials.

Activity fluctuations in these two aforementioned networks, the dorsal attention system and the default mode network, have been described to be intrinsically anticorrelated or rather to show the lowest degree of correlation of all resting-state networks (Fox et al., 2005, 2009; Fransson, 2005). Inverse correlations with performance across the two systems could then be ascribed to a source in merely one system and an epiphenomenon in the other. Visual inspection of the time courses renders this interpretation unlikely for our experiment. The prestimulus signal in the dorsal attention system that is associated with misses shows a slow build-up over time and that in the default mode network associated with hits shows a very brief build-up before stimulation, in each instance without mirroring signal behavior in the respective other system. And the evoked responses showed task-positive behavior in both the dorsal attention system and the precuneus, which also renders a hard-wired antagonistic relation unlikely.

Finally, in the intrinsic alertness system, hits were preceded by greater prestimulus activity than misses $\left(t_{(1,10)}=\right.$ $3.86, p<0.005)$. This pattern was consistent across all regions of this network (anterior thalamus: $t_{(1,10)}=3.76, p<0.005$; dorsal ACC: $t_{(1,10)}=3.6, p<0.005$ and trend in anterior insula: $t_{(1,10)}=2.23, p=$ $0.05)$. While the intrinsic alertness system is also in general found to be as much anticorrelated with the default mode network as the dorsal attention system (Fox et al., 2005; Fransson, 2005), its behavior was nonetheless opposite to the one in the dorsal attention system.

As in our previous studies, we performed a voxel-by-voxel mapping of the prestimulus effect by computing the contrast of the estimated signal at time point $0 \mathrm{~s}$ as a function of perceptual outcome (Fig. 5). This allowed investigating whether the effects observed in a priori defined ROIs were spatially specific to those regions. At the whole-brain mapping level, the prestimulus effect at time point $0 \mathrm{~s}$ on stimulus detection was significant $(p<0.05$ 


\section{A Default mode system}
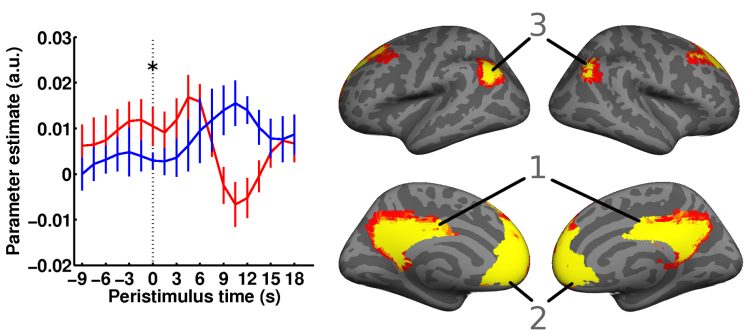

B Dorsal attention system
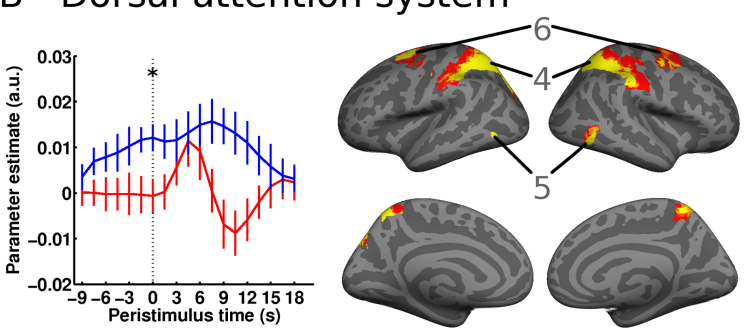

C Intrinsic alertness system
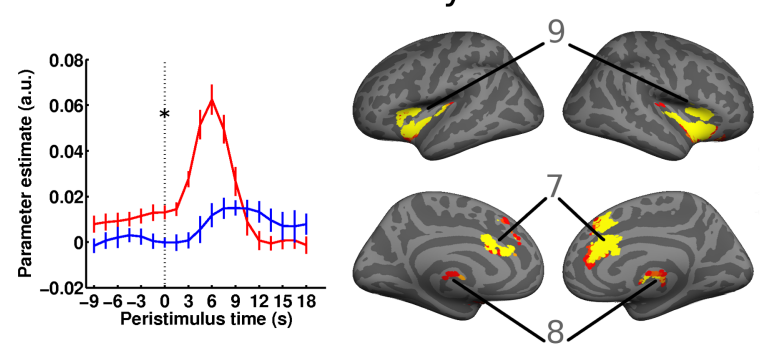

1 Precuneus/PCC
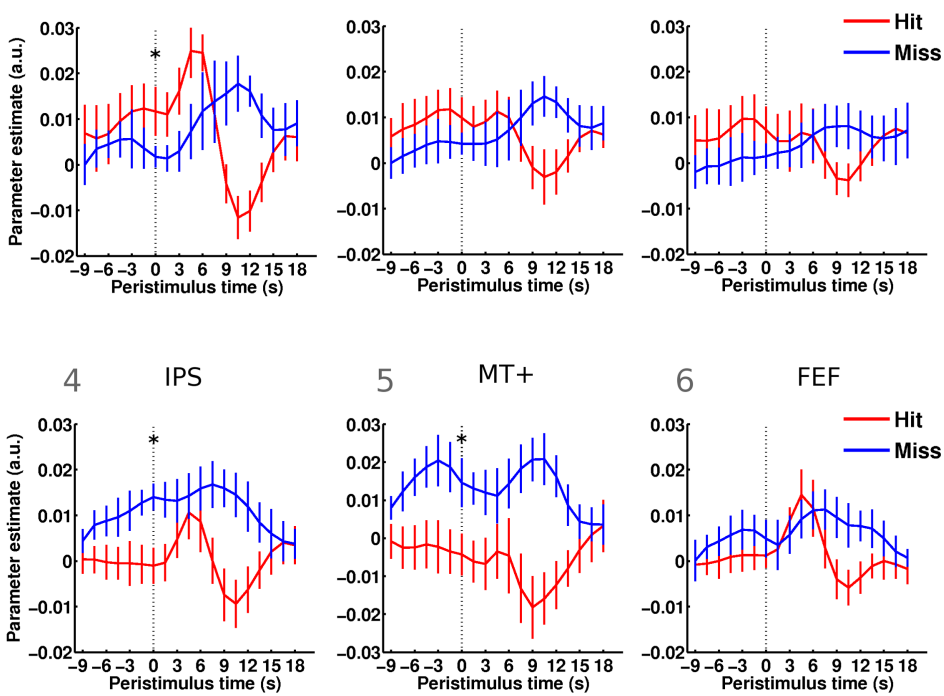

7 Dorsal ACC

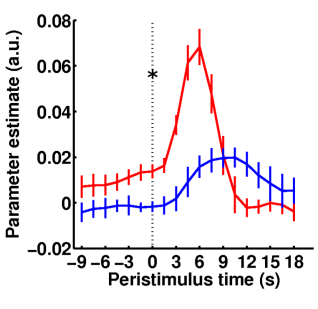

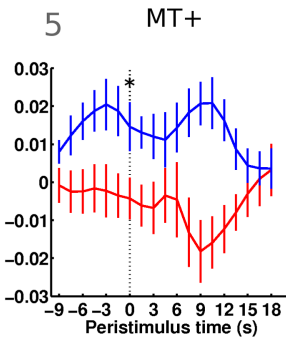

$2 \quad$ MPFC

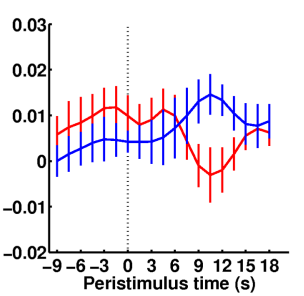

8 Anterior Thalamus

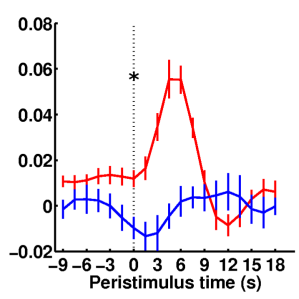

3 Lateral parietal

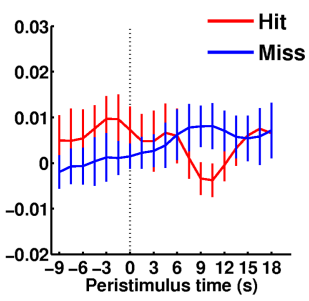

9 Anterior Insula

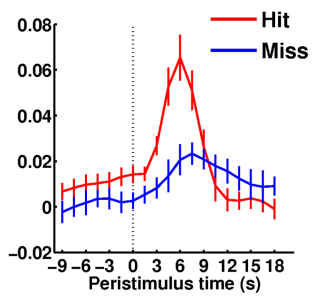

Figure 4. Prestimulus time courses from resting-state functional connectivity ( $\mathrm{rs}$-fc) networks. Left, Time courses averaged over the complete rs-fc systems. Middle, Rs-fc networks as defined by seed-based analysis of a resting-state scanning session. Numbers indicate regions of interest for which peristimulus activity time courses are plotted in the right-hand panels. Right, Time courses of individual regions of the respective network. While higher signal levels in the default mode system $(\boldsymbol{A})$ and the intrinsic alertness system $(\boldsymbol{C})$ were found before successful stimulus detection, higher signal in the dorsal attention system $(\boldsymbol{B})$ preceded misses. Asterisks indicate significant percept-dependent time course difference at time point 0 s. Error bars indicate \pm SEM.

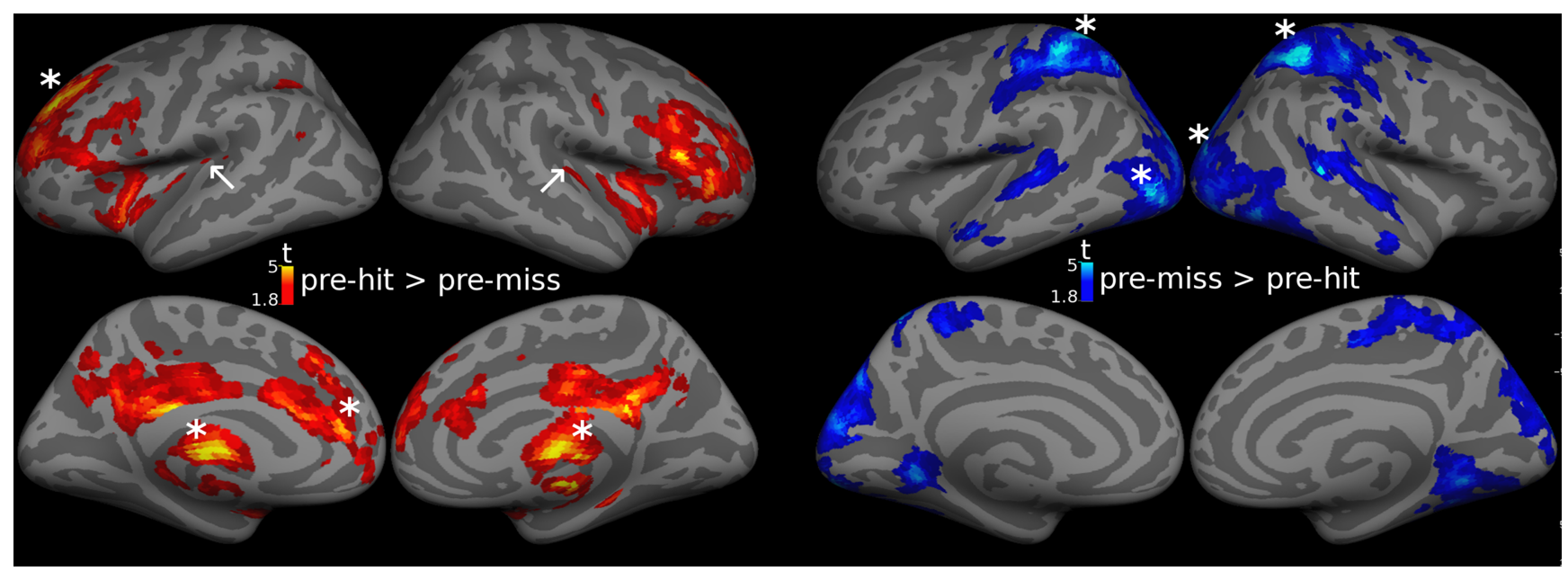

Figure 5. Statistical parametric maps of difference in prestimulus activity between hit and miss trials. Signal at time point $0 \mathrm{~s}$ (as estimated in the FIR-model, see Materials and Methods) was contrasted between hit and miss trials. The resulting second-level maps are shown at $p<0.05$ uncorrected to illustrate spatial specificity of prestimulus activity biasing for hits (left) and misses (right), respectively. Asterisks indicate regions with significant effects after correction for multiple comparisons. White arrows indicate Heschl's gyri. Signal time courses over the full peristimulus window are shown in Figures 3 and 4 for largely corresponding but independently defined ROls and rs-fc networks.

at cluster level after voxel-level threshold of $p<0.01$ and extent threshold $>50$ voxels) in the following regions: dorsal ACC [146 voxels, $(-15,39,3), z=3.71, p=0.034]$, an extended cluster [1064 voxels, $(27,0,12), z=4.35, p<0.001]$ containing thala- mus [left: $(-12,-21,12), z=4.27$; right: $(21,-15,15), z=4.04$ ] and left superior frontal gyrus [143 voxels, $-18,39,36), z=3.74$, $p=0.038]$ showed significantly higher prestimulus signal for hits than misses. Conversely, IPS [right: 477 voxels, $(30,-48,57), z=$ 
3.88, $p<0.001$; left: 347 voxels, $(-27,-45,54), z=3.82, p<$ 0.001 ] and posterior occipital cortex [left: 386 voxels, $(-27,-93$, $-18), z=3.49, p<0.001$; right: 367 voxels, $(15,-90,21), z=$ $3.34, p<0.001]$ showed higher prestimulus signal for misses than hits. The latter significant effects in the visual system extended beyond MT + to further regions of the dorsal visual system similar to descriptions of the dorsal attention system based on rs-fc (Fox et al., 2006).

\section{Discussion}

One popular and informative approach in studying the neural correlates of consciousness has been to compare evoked neural responses to perceived and nonperceived stimuli. As neural responses in themselves are sensitive to changes in stimulus properties, it is important that the correlation with conscious perception be assessed for identical stimuli. Several studies have evoked human brain responses and determined when and where stimulus-locked signal time courses show significant signal differences as a function of perceptual outcome (Ress et al., 2000; Pessoa and Padmala, 2005; Sergent et al., 2005; Pourtois et al., 2006). Instead of following the stimulus-evoked neural activity trace, however, one can ask what in turn may provide a neural source for this variability in neural responses to identical stimuli. Previous work has linked response variability to fluctuations in ongoing activity immediately before stimulation (Arieli et al., 1996).

Functional significance of prestimulus activity variations has been found in cued perceptual paradigms in nonhuman and human primates. In animal studies, effects on evoked responses (Arieli et al., 1996) and perceptual performance (Supèr et al., 2003) have been confined to a very short prestimulus time window, but the fMRI work in humans that relies on hemodynamic signals has uncovered effects with slower time constants (Sapir et al., 2005). While prestimulus effects in cued paradigms can be thought of as the variability in response to a cue the functional importance of slow spontaneous variations of ongoing activity has been shown in other studies. A prominent feature in ongoing electrical brain activity is the prevalence of oscillations that occur in various frequency bands. Over time, these different frequency bands display flexible adjustments in their phase synchrony as well as slow modulations in their overall power (Palva et al., 2005; Schroeder and Lakatos, 2009). Recordings of such modulations of large-scale oscillatory signals have been found to affect perceptual performance (Linkenkaer-Hansen et al., 2001; Monto et al., 2008). The precise relationship between the brain oscillations as well as slow cortical potentials and hemodynamic signals used in fMRI is not yet fully understood (He and Raichle, 2009), but the existing data suggest that the dynamics of ongoing activity are critical for the fate of incoming sensory information and include low frequency components (Buzsáki, 2006).

Following this line of thought, we have recently shown a biasing effect of fairly slow prestimulus activity variations onto subsequent perception of faces from an ambiguous figure (Hesselmann et al., 2008b) and of coherent motion in a moving dot kinematogram at threshold coherence (Hesselmann et al., 2008a). These effects were specific to the relevant sensory regions, namely the fusiform face area and the visual motion complex $(\mathrm{hMT}+)$, respectively. Here, we report a generalization of this finding beyond the visual domain to audition. In the auditory area responsive to our nearthreshold stimulus, we found that higher prestimulus activity biased toward detecting the stimulus, whereas low activity levels more likely yielded misses. This observation confirms our previous findings that ongoing activity levels in sensory areas impact on how several seconds later specifically related input will be perceived. As in our previous studies, this effect was rather weak potentially reflecting a truly small neuronal effect or alternatively sensitivity limitations of the neuroimaging technique. However, and different from our previous findings, the present study disclosed much stronger effects from ongoing activity in distributed functional networks that we had defined in an independent resting-state session.

We had anticipated such a difference because our present study involved detection of near-threshold stimuli in a freeresponse setting instead of two alternative forced choice decisions on ambiguous but clearly notable stimulation as in our earlier work. In accord with this view, evoked responses differed between hits and misses not only in auditory cortex but also in many other brain regions, likely reflecting all those processes that come into play once a stimulus will drive a behavioral response. Detailed discussion of these rather trivial effects is beyond the scope of our interest. One aspect of evoked responses, however, is relevant for our question. Peristimulus time courses of hit trials showed task-positive behavior in all three resting-state networks that we studied, including parts of the default mode network where the majority of paradigms elicit deactivation (Shulman et al., 1997; Binder et al., 1999; McKiernan et al., 2003). The latter network also showed the strongest inter-regional variation in that only the precuneus displayed a clear task-positive response that was followed by the same delayed deactivation as in the other constituent regions. Such a biphasic response pattern has previously been demonstrated in the precuneus in a memorydependent "transverse-patterning task" and related to associative memory processes (Meltzer et al., 2008).

The key issue of our study was to detect if and how baseline fluctuations in these resting-state networks bias detection of near-threshold auditory stimuli. Extending the observation of task-positive behavior in the precuneus, we found significantly higher activity in hit trials already before the evoked response. This observation may appear surprising, because previous work has associated activity levels in the precuneus with introspective, retrospective, or prospective mental processes (Buckner and Carroll, 2007; Mason et al., 2007; Botzung et al., 2008) and accordingly with deteriorated perceptual performance (Weissman et al., 2006; Boly et al., 2007; Eichele et al., 2008). It is important to note that those latter observations were made in paradigms in the somatosensory and visual modality that inevitably imposed a strong load on spatially selective attention. Conversely, in our study, discriminating a meaning-free broadband diotic stimulus from the continuous and qualitatively similar background (scanner) noise cannot be linked to fluctuations in spatial attention but rather to variations in the degree to which the mnemonic trace of this previously presented target stimulus was available to the subjects. As activity in the precuneus and adjacent retrosplenial and posterior cingulate cortex has been linked to retrieval success (Shannon and Buckner, 2004), we interpret our findings in the precuneus to indicate a facilitating effect of mnemonic mechanisms on recognition of the target stimulus. This interpretation is grounded in a view on ongoing activity as a carrier of dynamic predictions about future events (Fox and Raichle, 2007) and in line with our previous observations (Hesselmann et al., 2008a,b). A more general conclusion is that-as in other studies- the nature of ongoing activity is likely to reflect contextual effects, even a sparse paradigm being one of several relevant factors.

Further support for this interpretation comes from the findings we obtained in the dorsal spatial attention network and associated areas of the dorsal visual stream [even though the latter 
could also reflect intrinsic mechanisms of low-level cross-modal competition, see Laurienti et al. (2002)]. While generally associated with improved perceptual performance in paradigms requiring selective spatial attention (Burton and Sinclair, 2000; Corbetta and Shulman, 2002), activity in the dorsal attention system proved detrimental to stimulus detection in our paradigm, again presumably expressing the lack of spatial connotation in our stimulus and task. Our findings suggest that higher ongoing activity in the dorsal attention network favors the processing of spatial information and that this might compete with nonspatial attention for similar central resources. Interestingly, however, the dorsal attention system did show clear-cut taskpositive behavior in hit trials, i.e., evoked responses when stimuli were detected, which may reflect an automatic recruitment of spatial mechanisms by behaviorally relevant stimuli.

In the third resting-state network that we assessed and that we refer to as intrinsic alertness system, higher prestimulus activity level facilitated subsequent stimulus detection. Functionally, this effect in a paradigm which instructed subjects to continuously monitor auditory input for potential target occurrence, is readily related to mechanisms proposed in previous studies emphasizing task set maintenance (Dosenbach et al., 2007) or "salience" (or arousal) (Seeley et al., 2007). And similar to the network in those studies the one defined here also includes the anterior cingulate, anterior insula, and thalamic components. Together with the dorsal attention system and more ventral frontal and parietal regions, this network has been associated with "task-positive" behavior (Fox et al., 2005; Fransson, 2006). In our study, however, the effect of prestimulus signal in the intrinsic alertness network on stimulus detection was opposite in sign to that in the dorsal attention system and identical in sign to that from the default mode system. It hence appears likely that instead of a hard-wired generic relationship between these three networks, genuine and distinct effects of activity in all of them contributed independently to perceptual performance.

\section{Conclusion}

Together, our data contribute to a growing body of evidence that converges across species and signal modalities in linking variability of neural responses and behavioral performance to low frequency dynamics of ongoing brain activity. Our experiment illustrates that site and sign of these interactions depend on functional context. Beyond an expected local effect in accordingly specialized sensory cortex, we found performance in a freeresponse detection setting to be differentially affected by prestimulus activity levels in distributed functional networks. In contrast to previous related studies, higher prestimulus signal not only in the cingulo-insular intrinsic alertness system but also in the default mode network facilitated stimulus detection, whereas higher activity in the dorsal attention system was detrimental to perceptual performance. We hence conclude that the actual effects of ongoing activity fluctuations on processing subsequent stimuli do not only depend on the brain network where they occur but also on the functional context that is defined by the paradigm one uses to probe these effects. These findings speak against a simple dichotomic account of activity in these distributed networks and underline the flexibility with which their cooperation can change as a function of variable functional demands.

\section{References}

Arieli A, Sterkin A, Grinvald A, Aertsen A (1996) Dynamics of ongoing activity: explanation of the large variability in evoked cortical responses. Science 273:1868-1871.
Binder JR, Frost JA, Hammeke TA, Bellgowan PS, Rao SM, Cox RW (1999) Conceptual processing during the conscious resting state. A functional MRI study. J Cogn Neurosci 11:80-95.

Boly M, Balteau E, Schnakers C, Degueldre C, Moonen G, Luxen A, Phillips C, Peigneux P, Maquet P, Laureys S (2007) Baseline brain activity fluctuations predict somatosensory perception in humans. Proc Natl Acad Sci U S A 104:12187-12192.

Botzung A, Denkova E, Manning L (2008) Experiencing past and future personal events: functional neuroimaging evidence on the neural bases of mental time travel. Brain Cogn 66:202-212.

Buckner RL, Carroll DC (2007) Self-projection and the brain. Trends Cogn Sci 11:49-57.

Burton H, Sinclair RJ (2000) Attending to and remembering tactile stimuli: a review of brain imaging data and single-neuron responses. J Clin Neurophysiol 17:575-591.

Buzsáki G (2006) Rhythms of the brain. New York: Oxford UP.

Corbetta M, Shulman GL (2002) Control of goal-directed and stimulusdriven attention in the brain. Nat Rev Neurosci 3:201-215.

Dosenbach NU, Visscher KM, Palmer ED, Miezin FM, Wenger KK, Kang HC, Burgund ED, Grimes AL, Schlaggar BL, Petersen SE (2006) A core system for the implementation of task sets. Neuron 50:799-812.

Dosenbach NU, Fair DA, Miezin FM, Cohen AL, Wenger KK, ${ }^{* *}$ Dosenbach RA, Fox MD, Snyder AZ, Vincent JL, Raichle ME, Schlaggar BL, Petersen SE (2007) Distinct brain networks for adaptive and stable task control in humans. Proc Natl Acad Sci U S A 104:11073-11078.

Eckert MA, Menon V, Walczak A, Ahlstrom J, Denslow S, Horwitz A, Dubno JR (2009) At the heart of the ventral attention system: the right anterior insula. Hum Brain Mapp 30:2530-2541.

Eichele T, Debener S, Calhoun VD, Specht K, Engel AK, Hugdahl K, von Cramon DY, Ullsperger M (2008) Prediction of human errors by maladaptive changes in event-related brain networks. Proc Natl Acad Sci U S A 105:6173-6178.

Fox MD, Raichle ME (2007) Spontaneous fluctuations in brain activity observed with functional magnetic resonance imaging. Nat Rev Neurosci 8:700-711.

Fox MD, Snyder AZ, Vincent JL, Corbetta M, Van Essen DC, Raichle ME (2005) The human brain is intrinsically organized into dynamic, anticorrelated functional networks. Proc Natl Acad Sci U S A 102:9673-9678.

Fox MD, Corbetta M, Snyder AZ, Vincent JL, Raichle ME (2006) Spontaneous neuronal activity distinguishes human dorsal and ventral attention systems. Proc Natl Acad Sci U S A 103:10046-10051.

Fox MD, Snyder AZ, Vincent JL, Raichle ME (2007) Intrinsic fluctuations within cortical systems account for intertrial variability in human behavior. Neuron 56:171-184.

Fox MD, Zhang D, Snyder AZ, Raichle ME (2009) The global signal and observed anticorrelated resting state brain networks. J Neurophysiol 101:3270-3283.

Fransson P (2005) Spontaneous low-frequency BOLD signal fluctuations: an fMRI investigation of the resting-state default mode of brain function hypothesis. Hum Brain Mapp 26:15-29.

Fransson P (2006) How default is the default mode of brain function? Further evidence from intrinsic BOLD signal fluctuations. Neuropsychologia 44:2836-2845.

Golland Y, Bentin S, Gelbard H, Benjamini Y, Heller R, Nir Y, Hasson U, Malach R (2007) Extrinsic and intrinsic systems in the posterior cortex of the human brain revealed during natural sensory stimulation. Cereb Cortex 17:766-777

Greicius MD, Menon V (2004) Default-mode activity during a passive sensory task: uncoupled from deactivation but impacting activation. J Cogn Neurosci 16:1484-1492.

Gusnard DA, Raichle ME (2001) Searching for a baseline: functional imaging and the resting human brain. Nat Rev Neurosci 2:685-694.

He BJ, Raichle ME (2009) The fMRI signal, slow cortical potential and consciousness. Trends Cogn Sci 13:302-309.

Hesselmann G, Kell CA, Kleinschmidt A (2008a) Ongoing activity fluctuations in hMT + bias the perception of coherent visual motion. J Neurosci 28:14481-14485.

Hesselmann G, Kell CA, Eger E, Kleinschmidt A (2008b) Spontaneous local variations in ongoing neural activity bias perceptual decisions. Proc Natl Acad Sci U S A 105:10984-10989.

Laurienti PJ, Burdette JH, Wallace MT, Yen YF, Field AS, Stein BE (2002) 
Deactivation of sensory-specific cortex by cross-modal stimuli. J Cogn Neurosci 14:420-429.

Li CS, Yan P, Bergquist KL, Sinha R (2007) Greater activation of the "default" brain regions predicts stop signal errors. Neuroimage 38:640-648.

Linkenkaer-Hansen K, Nikouline VV, Palva JM, Ilmoniemi RJ (2001) Long-range temporal correlations and scaling behavior in human brain oscillations. J Neurosci 21:1370-1377.

Makeig S, Inlow M (1993) Lapses in alertness: coherence of fluctuations in performance and EEG spectrum. Electroencephalogr Clin Neurophysiol 86:23-35.

Mason MF, Norton MI, Van Horn JD, Wegner DM, Grafton ST, Macrae CN (2007) Wandering minds: the default network and stimulus-independent thought. Science 315:393-395.

McKiernan KA, Kaufman JN, Kucera-Thompson J, Binder JR (2003) A parametric manipulation of factors affecting task-induced deactivation in functional neuroimaging. J Cogn Neurosci 15:394-408.

Meltzer JA, Negishi M, Constable RT (2008) Biphasic hemodynamic responses influence deactivation and may mask activation in block-design fMRI paradigms. Hum Brain Mapp 29:385-399.

Monto S, Palva S, Voipio J, Palva JM (2008) Very slow EEG fluctuations predict the dynamics of stimulus detection and oscillation amplitudes in humans. J Neurosci 28:8268-8272.

Morosan P, Rademacher J, Schleicher A, Amunts K, Schormann T, Zilles K (2001) Human primary auditory cortex: cytoarchitectonic subdivisions and mapping into a spatial reference system. Neuroimage 13:684-701.

Palva JM, Palva S, Kaila K (2005) Phase synchrony among neuronal oscillations in the human cortex. J Neurosci 25:3962-3972.

Pessoa L, Padmala S (2005) Quantitative prediction of perceptual decisions during near-threshold fear detection. Proc Natl Acad Sci U S A 102: 5612-5617.

Pourtois G, De Pretto M, Hauert CA, Vuilleumier P (2006) Time course of brain activity during change blindness and change awareness: performance is predicted by neural events before change onset. J Cogn Neurosci $18: 2108-2129$.
Ress D, Backus BT, Heeger DJ (2000) Activity in primary visual cortex predicts performance in a visual detection task. Nat Neurosci 3:940-945.

Sapir A, d'Avossa G, McAvoy M, Shulman GL, Corbetta M (2005) Brain signals for spatial attention predict performance in a motion discrimination task. Proc Natl Acad Sci U S A 102:17810-17815.

Schroeder CE, Lakatos P (2009) Low-frequency neuronal oscillations as instruments of sensory selection. Trends Neurosci 32:9-18.

Scott SK, Johnsrude IS (2003) The neuroanatomical and functional organization of speech perception. Trends Neurosci 26:100-107.

Seeley WW, Menon V, Schatzberg AF, Keller J, Glover GH, Kenna H, Reiss AL, Greicius MD (2007) Dissociable intrinsic connectivity networks for salience processing and executive control. J Neurosci 27:2349-2356.

Sergent C, Baillet S, Dehaene S (2005) Timing of the brain events underlying access to consciousness during the attentional blink. Nat Neurosci 8:1391-1400.

Shannon BJ, Buckner RL (2004) Functional-anatomic correlates of memory retrieval that suggest nontraditional processing roles for multiple distinct regions within posterior parietal cortex. J Neurosci 24:10084-10092.

Shulman GL, Fiez JA, Corbetta M, Buckner RL, Miezin FM, Raichle ME, Petersen SE (1997) Common blood flow changes across visual tasks: II. Decreases in cerebral cortex. J Cogn Neurosci 9:648-663.

Singh KD, Fawcett IP (2008) Transient and linearly graded deactivation of the human default-mode network by a visual detection task. Neuroimage 41:100-112.

Sturm W, Longoni F, Fimm B, Dietrich T, Weis S, Kemna S, Herzog H, Willmes K (2004) Network for auditory intrinsic alertness: a PET study. Neuropsychologia 42:563-568.

Supèr H, van der Togt C, Spekreijse H, Lamme VA (2003) Internal state of monkey primary visual cortex (V1) predicts figure-ground perception. J Neurosci 23:3407-3414.

von Békésy G (1960) Experiments in hearing. New York: McGraw-Hill.

Weissman DH, Roberts KC, Visscher KM, Woldorff MG (2006) The neural bases of momentary lapses in attention. Nat Neurosci 9:971-978. 